\title{
Biopsy-Proven Cardiomyopathy in Heterozygous Fabry's Disease
}

\author{
Norimichi Koitabashi, MD; Toshihiro Utsugi, MD; \\ Ryotaro Seki, MD; Eiichi Okamoto, MD; Yoshichika Sando, MD; \\ Yoshiaki Kaneko, MD; Ryozo Nagai, MD
}

\begin{abstract}
A 23-year-old woman with heterozygous Fabry's disease who had acroparesthesia was admitted to hospital for precise examination of the disease before childbearing. She had no cardiac-related symptoms and no abnormality on physical examination. The a-galactosidase A activity in her leukocytes was present, but lower than normal. However, the endomyocardial biopsy showed specific changes for Fabry's disease. As Fabry's disease is a rare $\mathrm{X}$-linked recessive inborn error of glycosphingolipid metabolism, heterozygous females are usually asymptomatic, but rarely can be affected as severely as hemizygous males. This is an isolated case of heterozygous Fabry's disease in a female in whom cardiac involvement was detected by endomyocardial biopsy, although she had no cardiac abnormality on physiological examinations. In conclusion, endomyocardial biopsy is useful for evaluation of the cardiac involvement of Fabry's disease even in an asymptomatic case. (Jpn Circ J 1999; 63: 572-575)
\end{abstract}

Key Words: Fabry's disease; Heterozygote; Cardiac biopsy; Cardiac manifestation

$\mathbf{F}$ abry's disease is a rare $\mathrm{X}$-linked recessive inborn error of glycosphingolipid metabolism. The deficient activity of the enzyme a-galactosidase A results in accumulation of a neutral glycosphingolipid, globotriaosylceramide, primarily in vascular endothelial lysosomes as well as in lysosomes in the kidneys, heart valves and cardiac muscle. In classically affected hemizygous males, the major disease manifestations include angiokeratoma, corneal dystrophy, acroparesthesias, and vascular disease of the heart, kidneys and brain, leading to early death in adulthood. However, heterozygous females have a wide range of clinical manifestations, ranging from asymptomatic to moderate or severe disease. Because random inactivation of the $\mathrm{X}$ chromosome occurs during early embryogenesis in females, they exhibit a mosaic pattern of expression of X-linked genes! Some heterozygotes have cardiac involvement in adulthood, such as mitral valve prolapse, left ventricular hypertrophy and arrhythmia ${ }^{2-6}$ We present a female case of Fabry's disease in which cardiomyopathy was proven by endomyocardial biopsy.

\section{Case Report}

A 23-year-old woman was admitted to hospital for precise evaluation of Fabry's disease before her pregnancy. Thirteen years earlier, she had been diagnosed as having Fabry's disease from measurement of a -galactosidase activity of her white blood cells. She had been taking carbamazepine for the pain and numbness of her distal extremities since she was 8 year old. The crises of pain

(Received November 9, 1998; revised manuscript received April 9, 1999; accepted April 14, 1999)

Second Department of Internal Medicine and Health Science, Gunma University, School of Medicine, Maebashi, Japan

Mailing address: Norimichi Koitabashi, MD, Division of Cardiology, Gunma Prefectural Cardiovascular Center, 3-12 Kameizumi-machi, Maebashi-city, Gunma-pref 371-0004, Japan were caused by fever and fatigue. There was no family history of cardiovascular, renal or neurological disorders.

On admission, small red rashes were observed on her forehead and back. Her neurological findings were almost normal, except for pain of the terminal limbs. The whorllike corneal opacity, which is unique to Fabry's disease, was observed by slit-lamp microscopy! The results of routine hematological, chemical, and urine tests were

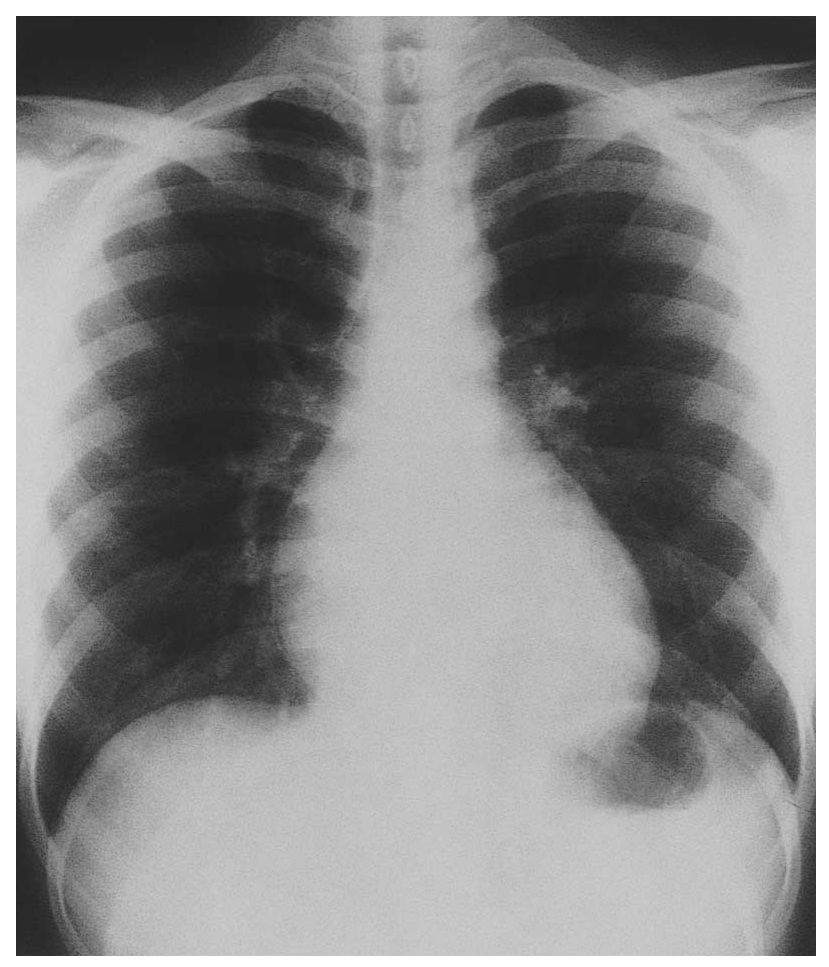

Fig 1. Chest radiograph on admission showing a slightly increased cardiothoracic ratio of $52 \%$, without pulmonary congestion. 


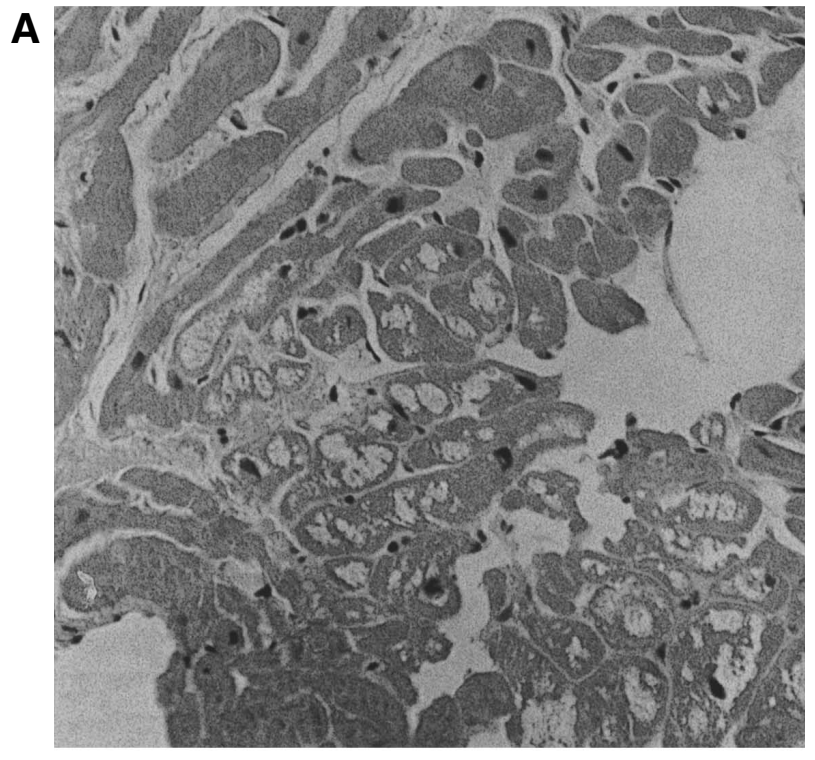

B

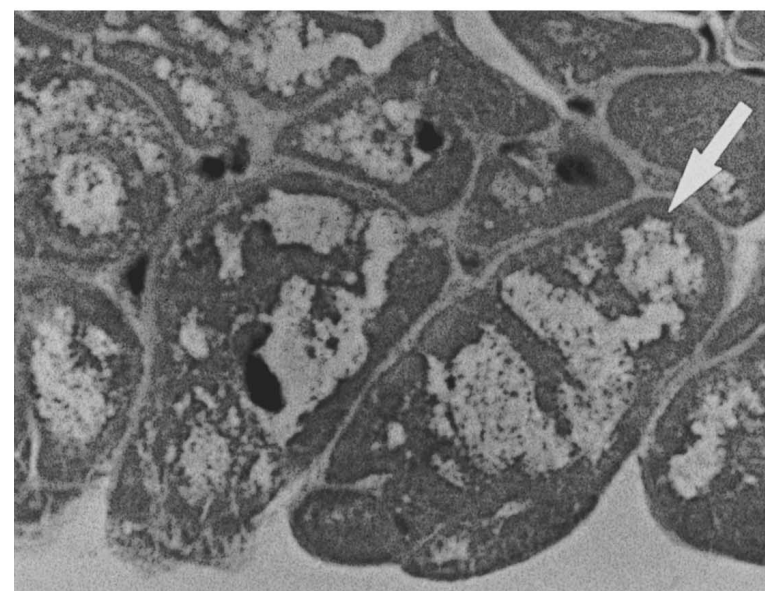

Fig 2. Endomyocardial biopsy specimens show sarcoplasmic vacuolization (arrow), leaving large clear spaces. (A) $\times 400$, (B) $\times 1000$ (hematoxylin-eosin).

Table 1 Hemodynamic Data From Cardiac Catheterization

\begin{tabular}{lc}
\hline \hline Pressure measurements $(\mathrm{mmHg}$, systole/diastole) & \\
Pulmonary capillary wedge & $11^{*}$ \\
Pulmonary artery & $23 / 13$ \\
Right ventricle & $29 / 7$ \\
Right atrium & $5 *$ \\
Left ventricle & $104 / 15$ \\
Central aorta & $118 / 76$ \\
Cardiac output $(\text { L/min })^{\S}$ & 5.5 \\
Cardiac index $\left(L \mathrm{~min}^{-1} \mathrm{~mm}^{-2}\right)^{\S}$ & 3.4 \\
Left ventricular ejection fraction $(\%)^{\pi}$ & 61 \\
\hline
\end{tabular}

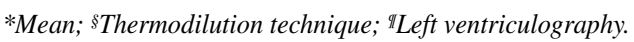

normal. The chest radiograph showed a slightly increased cardiothoracic ratio of 52\%, without pulmonary congestion (Fig 1). Echocardiography demonstrated that the valves, cavity size, wall thickness and contractilities were normal. A 12-lead electrocardiogram was normal. Hera-galactosidase activity in the white blood cells was $39 \mathrm{nmol} \cdot \mathrm{mg}$ of $\operatorname{protein}^{-1} \cdot \mathrm{h}^{-1}$, which was lower than that of the normal control $\left(67-116 \mathrm{nmol} \cdot \mathrm{mg}\right.$ of protein $\left.{ }^{-1} \cdot \mathrm{h}^{-1}\right)$, but her plasma a-galactosidase activity was normal. Her grandmother,
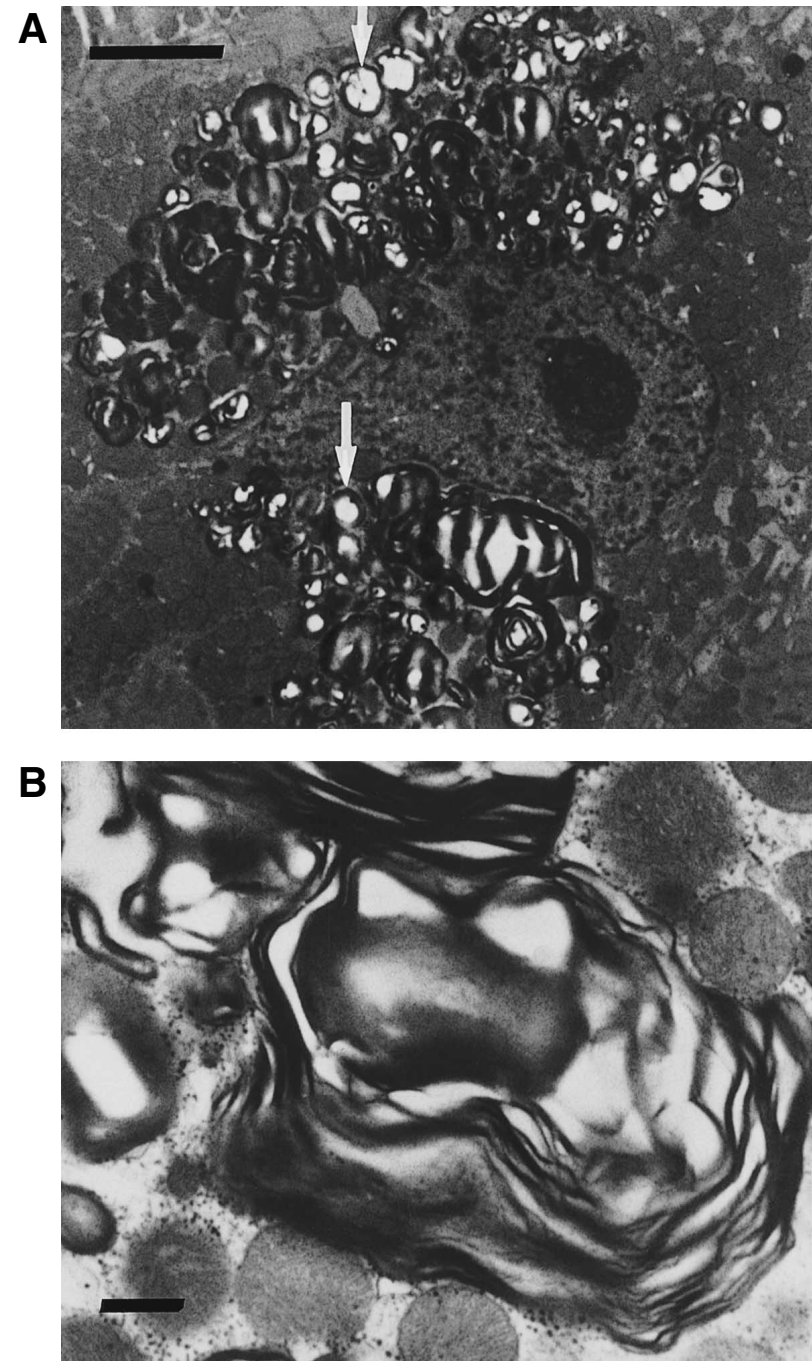

Fig 3. Electron microscopy of the myocardial cell shows perinuclear typical lysosomal inclusions (A: arrows) with a concentric lamellar configuration (B). The black bar indicates $5 \mu \mathrm{m}$ in $\mathrm{A}$, and $1 \mu \mathrm{m}$ in B.

parents and sister had no remarkable abnormalities of enzyme activities. A skin biopsy performed from the small red rash on her back showed nonspecific telangiectasias. Because the patient required precise examination before pregnancy, we performed cardiac catheterization with her written informed consent. Endomyocardial biopsy was necessary to confirm the diagnosis of Fabry's disease because the enzyme assays had showed different results between the white blood cells and the plasma. The hemodynamic data were within normal range, and left ventriculography was normal (Table 1). A percutaneous endomyocardial biopsy was performed from the left ventricle, and the cardiac specimens were stained with hematoxylin-eosin (Fig 2A, B). Approximately $30 \%$ of the myocardial cells revealed marked sarcoplasmic vacuolization. On electron microscopy (Fig 3A, B), lysosomal inclusions with concentric lamellar configuration were demonstrated. These findings were common in Fabry's disease, so she was diagnosed as an isolated case of heterozygous Fabry's disease and her organopathy due to this disease were acroparesthesia and mild cardiomyopathy. On the basis of these results, we suggested she have careful medical observation whilst pregnant. 


\section{Discussion}

We have described a case of heterozygous Fabry's disease in which cardiomyopathy was proven by endomyocardial biopsy. As reported in the heterozygotes, she had minor symptoms of the disease, such as acroparesthesia, corneal opacities, and skin lesions, but no obvious cardiac manifestation and her cardiac function was almost normal. The only sign suggesting myocardial abnormality was the slight cardiomegaly on chest radiograph. The myocardial biopsy specimen, however, showed an obvious abnormality for Fabry's disease.

Fabry's disease is an X-linked recessive pleiotropic genetic disorder. Deficiency of the lysosomal enzyme agalactosidase results in accumulation of a neutral glycosphingolipid, chiefly globotriaosylceramide. The gene for this enzyme has been mapped to the middle of the long arm of the X chromosome. As might be expected with a slowly progressive storage disorder, the clinical manifestations are protean and nonspecific, so it is not too surprising that the correct diagnosis is often delayed or missed. In childhood the disease is characterized by the appearance of ectatic vascular skin lesions in the lower abdomen and groin, episodic pain most commonly affecting the limbs, and a subtle and symptomless corneal dystrophy. In adult males virtually no system is spared, with vascular disease of the kidneys, heart, and brain contributing to progressive disability. Without dialysis or transplantation most hemizygous males will succumb to renal failure by the age of 50 ! The cardiac involvement, such as congestive heart failure, cardiomegaly, arrhythmias, mitral insufficiency (especially mitral valve prolapse), aortic stenosis and myocardial ischemia-infarction in affected middle-aged males has been described ${ }^{1-3,7-9}$ Recently, an atypical variant of Fabry's disease, with clinical manifestations limited to the heart, was found with a high frequency among Japanese males with left ventricular hypertrophy! ${ }^{10}$

Heterozygous females of 'classical' Fabry's disease have a wide range of clinical manifestations, ranging from asymptomatic to moderate to severe. Because inactivation of X chromosome during early embryogenesis occurs at random and is maintained in all daughter cells, females exhibit a mosaic pattern of expression of X-linked genes!,11 Approximately $30 \%$ of heterozygotes have a few, isolated skin lesions, less than $10 \%$ have acroparesthesias, and about $70 \%$ have the whorl-like corneal dystrophy! Some heterozygotes have an associated heart disorder in adulthood; mitral valve prolapse, left ventricular hypertrophy and arrhythmia have been reported ${ }^{2-6}$ The diagnosis of heterozygotes has been attempted by measuring the $\alpha$ galactosidase activity in leukocytes, plasma, fibroblasts and hair follicles; however, this is not necessarily reliable because the enzyme activity varies markedly in heterozygotes due to the random inactivation of the $\mathrm{X}$ chromosome, ${ }^{1,6}$ as was seen in the present case with the discrepancy between the leukocyte and plasma $\alpha$-galactosidase activity. Thus, the diagnosis of heterozygous Fabry's disease, especially in an isolated case, is sometimes difficult by means of the enzyme assay.

Percutaneous endomyocardial biopsy with electron microscopic analysis appears to be a useful way to diagnose and evaluate the severity of cardiac involvement in Fabry's disease $!^{2}$ Endomyocardial biopsy from patients with Fabry's disease typically shows a marked perinuclear vacuolization, which is the glycosphingolipid leached out by standard paraffin processing, and electron microscopy reveals numerous cytoplasmic inclusion bodies containing glycolipid showing a concentric lamellar configuration, $1,2,12$ Similar lamellar inclusion bodies occur in Tay-Sachs disease, fucosidosis, and Niemann-Pick disease, but they have different periodicity and the clinical manifestations are distinct ${ }^{13,14}$ Recently, immunofluorescence analysis of globotriaosylceramide has been reported for detecting the cardiac involvement of the heterozygote. ${ }^{6}$

Generally, it has been described that less than $1 \%$ of cases of heterozygous Fabry's disease has cardiac involvement. Most cases of heterozygous Fabry's disease who have had endomyocardial biopsy performed have cardiacrelated symptoms and/or manifest an abnormality on electrocardiography and echocardiography. Previous reports have suggested that not only hemizygotes but also heterozygous females are rarely progressive or reveal cardiac manifestation. Sudden death in an elderly heterozygote has been reported, 14 In the present case, the precise evaluation of cardiac function was necessary to prepare for childbearing.

We suggest that endomyocardial biopsy may be a useful method for confirming the diagnosis of Fabry's disease for heterozygous patients without a family history, even if they do not show a cardiac-related abnormality in physiological examination. The evaluation of myocardial manifestation by histological methods could be useful for understanding the severity of heterozygous Fabry's disease.

\section{Acknowledgments}

We thank Drs Kohji Itoh and Hitoshi Sakuraba (Department of Clinical Genetics, Tokyo Metropolitan Institute of Medical Science) for the enzyme assay ofa -galactosidase activity and for their critical support.

\section{References}

1. Desnick RJ, Ioannou YA, Eng CM: a-Galactosidase A deficiency: Fabry disease. In: Scriver CR, Beaudet AI, Sly WS, Valle D, editors. The metabolic and molecular bases of inherited disease, Vol. 7. New York: McGraw-Hill, 1995: 2741-2784

2. Sakuraba H, Yanagisawa Y, Igarashi T, Suzuki Y, Suzuki T, Watanabe K, et al: Cardiovascular manifestations in Fabry's disease: a high incidence of mitral valve prolapse in hemizygotes and heterozygotes. Clin Genet 1986; 29: 276-283

3. Goldman ME, Cantor R, Schwartz MF, Baker M, Desnick RJ: Echocardiographic abnormalities and disease severity in Fabry's disease. J Am Coll Cardiol 1986; 7: 1157-1161

4. Yokoyama A, Yamazoe M, Shibata A: A case of heterozygous Fabry's disease with PR interval and giant negative T waves. $\mathrm{Br}$ Heart J 1987; 57: 296-299

5. Hillsley RE, Hernandez E, Steenbergen C, Bashore TM, Harrison $\mathrm{JK}$ : Inherited restrictive cardiomyopathy in a 74-year-old woman: a case of Fabry's disease. Am Heart J 1995; 129: 199-202

6. Ito K, Takenaka T, Nakao S, Setoguchi M, Tanaka H, Suzuki T, et al: Immunofluorescence analysis of trihexosylceramide accumulated in the hearts of variant hemizygotes and heterozygotes with Fabry disease. Am J Cardiol 1996; 78: 116-117

7. Suzuki M, Goto T, Kato R, Yamauchi K, Hayashi H: Combined atrioventricular block and sinus node dysfunction in Fabry's disease. Am Heart J 1990; 120: 438-440

8. Pochis WT, Litzow JT, King BG, Kenny D: Electrophysiologic findings in Fabry's disease with a short PR interval. Am J Cardiol 1994; 74: $203-204$

9. Yamaguchi T, Kuwako K, Ikari Y: Fabry's disease with complete atrioventricular block: histological evidence of involvement of the conduction system. Br Heart J 1992; 68: 323-325

10. Nakao S, Takenaka T, Maeda M, Kodama C, Tanaka A, Tahara M, et al: An atypical variant of Fabry's disease in men with left ventricular hypertrophy. N Engl J Med 1995; 333: 288-293

11. Redonnet-Vernhet I, van Amstel KP, Jansen RPM, Wevers RA, Salvayre R, Levade T: Uneven X activation in a female monozygotic twin pair with Fabry disease and discordant expression of a novel 
mutation in thea-galactosidase A gene. J Med Genet 1996; 33: $682-$ 688

12. Fowles RE, Mason JW: Role of cardiac biopsy in the diagnosis and management of cardiac disease. Prog Cardiovasc Dis 1984; 27: $153-172$
13. Ferrans VJ, Hibbs RG, Burda CD: The heart in Fabry's disease: A histochemical and electron microscopic study. Am J Cardiol 1969; 24: $95-110$

14. Carter N, Milroy CM, Shepherd RT: Sudden death in elderly women with Fabry's disease. Am J Forensic Med Pathol 1995; 16: 21-26 